\title{
The SEP-Approach to Evaluate Large-Scale Intelligent Freight Transport Systems
}

\author{
Corné Versteegt, Alexander Verbraeck \\ Delft University of Technology \\ Faculty of Technology, Policy and Management \\ Systems Engineering Group \\ P.O. Box 5015, 2600GA Delft, The Netherlands \\ E-Mail: \{cornev, alexandv\}@tbm.tudelft.nl
}

\begin{abstract}
In this paper we present the SEP-approach, an approach to evaluate intelligent logistic systems and their control systems. The SEP-approach consists of four phases in which three types of models are used; Simulation, Emulation, and Prototypes, of control systems and logistic resources. In the first phase a simulation model is made of the physical logistic processes. A second simulation model is made containing the logic of the control systems. This simulated control system is used to control different models of the logistic resources throughout the entire SEP-approach. In the second phase emulation models are made of the logistic resources. The emulated logistic resources are controlled by the simulated control system. In the third phase the simulated control system controls prototypes of logistic resources. In the fourth and final phase combinations of simulated, emulated, and prototype logistic resources are used simultaneously, all controlled by the simulated control system. This provides a 'rich' test environment in which several aspects of the automated freight transport system can be studied, e.g. communication equipment, obstacle sensors, scheduling and routing of AGVs, and safety control systems. The SEP approach was successfully applied to evaluate Automated Guided Vehicles and material handling stations for the Underground Logistic System Schiphol. The ULS Schiphol is future large-scale automated logistic system in the Netherlands. By applying the SEPapproach the control systems and logistic resource designed for ULS Schiphol were evaluated before implementation and commissioning.
\end{abstract}

Keywords: Simulation, Emulation, Prototyping, Testing, Automated logistic systems.

\section{Introduction}

Automation of resources for transport, transshipment, and storage is an important development in modern logistic systems. The logistic resources become more and more automated, like Automated Guided Vehicles. Some automated logis tic systems are already operational, such systems use small numbers of logistic resources, the transport distances are short, and human intervention still plays an important role (Müller 1983). In this research the focus is on large-scale automated freight transport systems, such systems use large numbers of automated logistic resources and the control over the resources is fully automated.

Control systems for logistic and transport systems are among the most complex control systems in existence (Pyle et al. 1993). Such control systems have to control many concurrent processes, have to react to input within strict time windows, have a distributed nature, and have to work with large sets of heterogeneous data. At this moment control systems of automated logis tic systems are only fully tested after commissioning at the shop floor (Schiess 2001, Auinger et al. 1999). It is difficult to test or pre-commission a control system before implementing and coupling control systems with the real systems-beingcontrolled. The testing takes place during the startup phase of the system-being-controlled. This is an expensive, risky and error-prone way of developing control systems (McGregor 2002).

Simulation has often been used to test control systems and automated logistic systems (Meer 2001, Banks 1998). Simulation, however, has certain limitations for testing automated logistic systems. Simulation does not offer possibilities for detailed studies of communication aspects and technical aspects. Large-scale automated systems use new technologies that not have been proven in practice. To evaluate automated logistic systems and their control systems a 'richer' set of modeling techniques is needed.

Auinger et al. (1999) state that it is vital to test control systems before implementing them. They suggest using combinations of reality and simulation to test control systems. Four possible approaches to test control systems can be distinguished, see Figure 2 (Auinger et al. 1999).

a. The traditional way to test control systems. A combination of a control system and logistic system in reality. The control system is tested after commissioning. 
b. Soft commissioning. A combination of a control system in reality and a simulated logistic system. This step is also called emulation (Schiess 2001, Mueller 2001).

c. Reality in the loop. A combination of a simulated control system and a real logistic system. This phase is also called simulation for real-time control (Versteegt \& Verbraeck 2002b).

d. Off-line simulation. A combination of a simulated control system and a simulated logistic system.

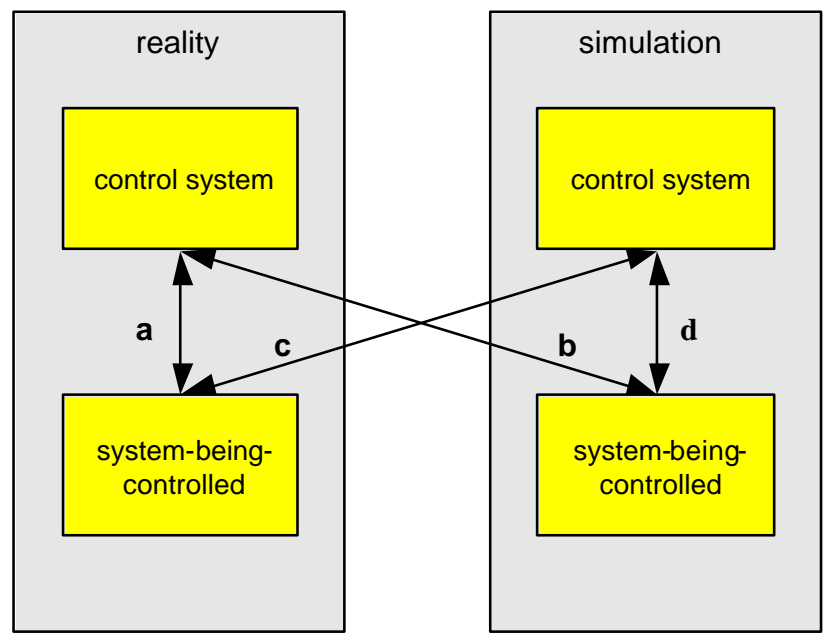

Figure 1. Approaches to test control systems

At this moment control systems are only fully tested after commissioning at the shop floor, combination a in Figure 1. In this paper we introduce an approach to evaluate control systems and automated logistic systems before commissioning. The approach, called SEPapproach, uses combinations of Simulation, Emulation, and Prototyping, of control systems and logistic systems being-controlled.

\section{SEP approach}

The SEP-approach consists of four phases in which three types of models are used, as can be seen in Figure 2 .

1. Off-line simulation. In the first step simulation models are constructed of the control system and the logistic system-being-controlled. The primary goal of this phase is to test the control logic and the assumptions on the logistic system. The control system and system-being-controlled are first modeled in one simulation model. When the control logic and assumptions on the logistic system have been tested they can be separated in different simulation models. The simulated control system is used throughout the entire SEP-approach.
2. Emulation of logistic resources. In this phase the simulated control system developed in the first phase controls emulated logistic resources. The emulation models are highly detailed simulation models of the logistic resources. Although these models are still simulation models, they represent the real physical logistic resources much more closely. For example, emulation models of AGVs contain the actual software of the on-board control systems of AGVs. The goal of this phase is to test the communication between control system and logistic system and debug software within the logistic resources.

3. Prototyping of logistic resources. In this phase the simulated control system is used to control prototype logistic resources. These prototypes can be scale models or first implementations of AGV or material handling stations. The primary goal of this phase is to test the technical aspects, like vehicle dynamics. We use scale models and prototypes since the real logistic resources are not yet available.

4. Combining simulation, emulation, and prototypes. In this phase the models we combine the simulation models that were developed in first phase, the emulation models from the second phase, and the prototypes from the third phase. All three different types of logistic resources are controlled by the same simulated control system.

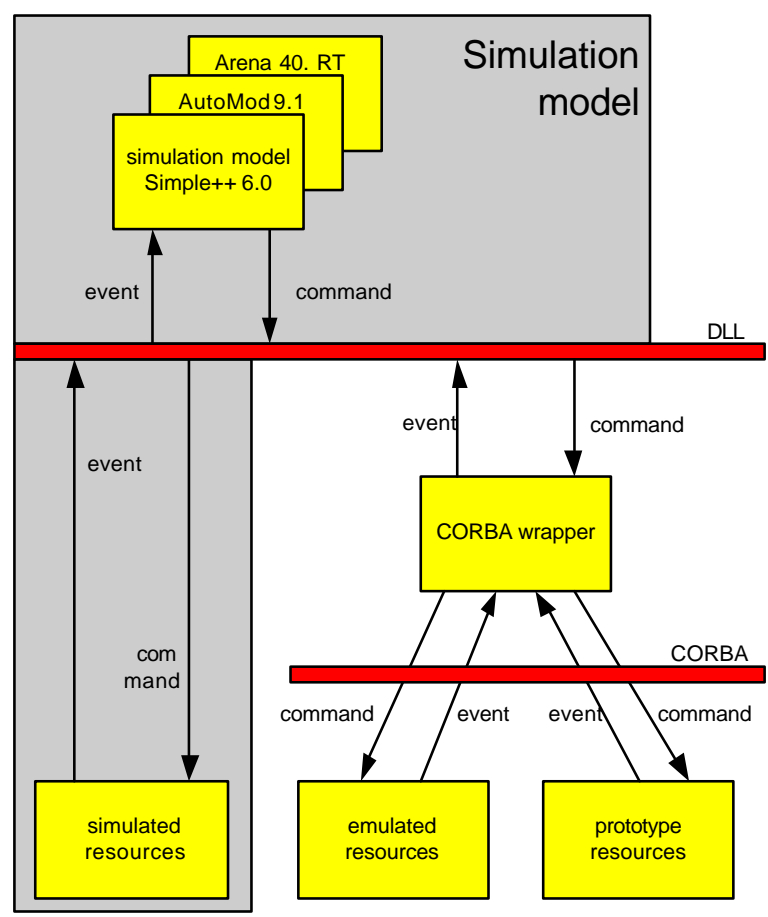

Figure 2. SEP-approach to evaluate control systems and automated logistic systems 
The SEP-approach combines combinations $\mathrm{c}$ and $\mathrm{d}$ from Figure 1

\section{Communication}

An important part of the SEP-approach is the communication between control system and logistic resources. The communication has to be independent of hardware and software, each control system and logistic resources uses other technologies and software.

The communication within the SEP-approach has an asynchronous command/event-based structure. Two possible forms of communication exist to send information from one process to another process, synchronous and asynchronous communication (Ben-Ari 1990). In synchronous communication the processes between which information is exchanged have to synchronize. The information is transferred when one process is ready to send and the other process is ready to receive the information (Ben-Ari 1990). Within large-scale automated logistic systems many control and physical processes take place. Synchronous communication can easily lead to nervous behavior of the system-being-controlled. When a control process is not ready to communicate, the physical logistic process is delayed, e.g. an AGV has to wait before it can start driving. These delays are caused by the lack of flexibility of synchronous communication and the speed differences between control processes and physical processes. A more flexible solution is asynchronous communication. Here a buffer is introduced in order to allow asynchronous communication (Ben-Ari 1990). This buffer is a queue of messages. Messages from the sending process are put in the queue, while the receiving process retrieves messages from the queue. Asynchronous communication is based on the principle of 'send-andforget' and a mailbox (Verbraeck \& Versteegt 2001). The sending process sends a message to the mailbox, forgets about it and continues to operate normally.

Commands are send top-down from the control systems to the logistic resources. When the logistic resources have reached a certain state in executing commands they generate events and send these events, or eventnotification, back to the control system. The control system can then decide the next command that is needed.

\section{Application of the SEP approach}

The SEP approach was applied to evaluate designs of AGVs and automated material handling systems for the Underground Logistic System Schiphol. The ULS Schiphol is a future large-scale automated logistic system near Amsterdam Airport Schiphol in the Netherlands (Versteegt \& Verbraeck 2002a). The SEP approach was applied, since the ULS Schiphol differs from normal AGVbased systems (Pielage 2001, 2000). The ULS Schiphol is a large-scale automated logistic system. It uses large numbers of AGVs, up-to 400. The available space, however, is very limited, since the system is mostly underground. The available space for carrying out the logistic operations is very small. Controlling AGVs, deadlock avoidance, and collision avoidance are critical. The distances between terminals are larger than in other AGV-based systems. The cargo units that are transported also differ from other AGV-based systems. The ULS Schiphol will transport main-deck airfreight pallets and flower cars. The dimensions and characteristics of these cargo units differ from other AGV-based systems. To cope with these new and different demands new technologies for AGVs and material handling stations were developed. The SEP approach was used to evaluate the control systems, AGVs and material handling stations designed for the ULS Schiphol.

\subsection{Simulation}

In the first phase the control systems, AGVs, and automated material handling systems were modeled in three simulation packages; Simple++ version 6.0 (Aesop 1999), Arena version 4.0 RT (Kelton, Sadowski \& Sadowski 1998), and AutoMod version 9.1 (Banks 2000). We wanted to show that the SEP-approach is independent of software platforms. The control logic was tested in fully simulated environment. The testing could be carried out faster than real-time. This is very powerful to trace failures that do not occur on regular basis, like deadlocks between AGVs. The simulation environment also provided a safe environment and good control over the experiments.

\subsection{Emulation}

In the second phase emulation models were developed of the AGVs. These are highly detailed simulation models of the AGVs. They contain the actual control software of the prototype AGVs. Their behavior represents the behavior of real AGVs very closely. The goal of this phase was to check the AGV-software for programming errors and to test the communication between AGVs and control system.

The ULS Schiphol is an underground automated logistic system. Within tunnels communication with the AGVs can be difficult (Verbraeck \& Versteegt 2001). Reducing the need for communication in the tunnels reduces the need for special and expensive communication equipment. It was, therefore, important to minimize communication between control systems and logistic resources.

The communication was asynchronous and command/event-based. Two types of commands and five types of events were used. The simulated control system sends commands to the AGVs.

- AGV-Init. The AGVInit command initializes an $\mathrm{AGV}$ on an initial position and an initial 
orientation. There are no differences between simulated, emulated, and prototypes AGVs. The AGV-Init has the following attributes; AGVname, X-begin, Y-begin, and O-begin. This command is executed only once for every AGV that enters an experiment.

- AGV-Exec. The second command, AGVExec, is an execution command. It is used to give an AGV a command to drive toward a certain destination. The AGV-Exec command has the following attributes; AGV-name, Track-name, Xdestination, Y-destination, O-destination. This command is repeated every time an AGV is send to a new destination.

AGVs send events, or event notifications, to the simulated control system when they have reached certain stages in executing the commands received from the control system. These events 'trigger' the simulated control system to make control decisions and are used to synchronize the position of the AGVs and 'virtual' position of the AGV in the simulated control system. The AGV use four event that are related to track which they follow, as can be seen in Figure 3.

- ON: an event to indicate that the front of the vehicle has entered the track. The track is now occupied.

- NEAR: a so-called 'near-event' to the simulation model when they have 'neared' the end of a track.

- POSITIONED: the front of the vehicle is at the end of the virtual track. When the vehicle has to drive to a destination, indicated by its front, we call the vehicle 'positioned', and actions like loading or unloading can start.

- PASSED: the passed event is given when the vehicle left the track entirely, i.e. the back of the vehicle passed the end of the track. For safety systems this is an indication that the track is 'clear'.

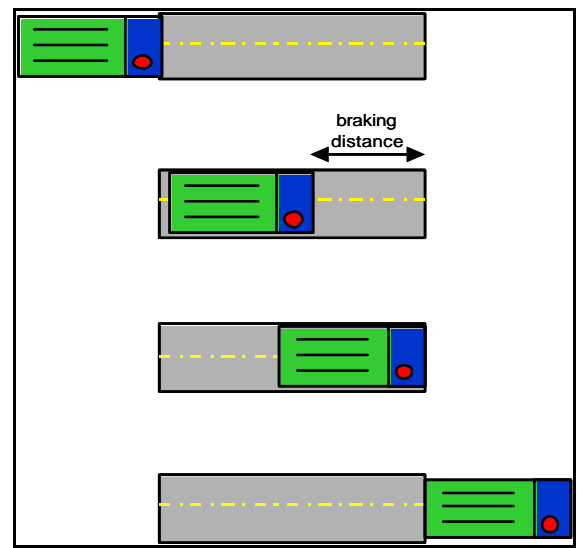

Figure 3. Synchronization of place: on-event, near-event, positioned-event, and passed-event
Besides the four events that are used to signal the control system that a certain stage in the execution of a command has been reached, a time-out event was introduced. The time-out event is generated when an event is expected but it is not generated. For example, when an $\mathrm{AGV}$ is broken down, it cannot generate an event. After a certain amount of time a time-out event is generated to inform the control system that the command has not been executed.

\subsection{Prototyping}

In third phase prototype AGVs and material handling stations were controlled by the simulated control system. The prototyping took place at the TestSite, a transportation research center in the Netherlands. The TestSite is equipped with a fleet of 2 meter long 1:3 scale models and 6 meter long 1:1 prototype AGVs (Versteegt \& Verbraeck 2002a). The TestSite has automated material handling stations with PLCs to control them, a wireless network, and an operator control room with several computers systems. The TestSite has a floor area of 1,600 square meters, and it has a grid of magnets for calibrating purposes of the vehicles. On this floor, a complex set of virtual tracks with crossings can be created to test the AGVs, material handling stations, and their control systems.
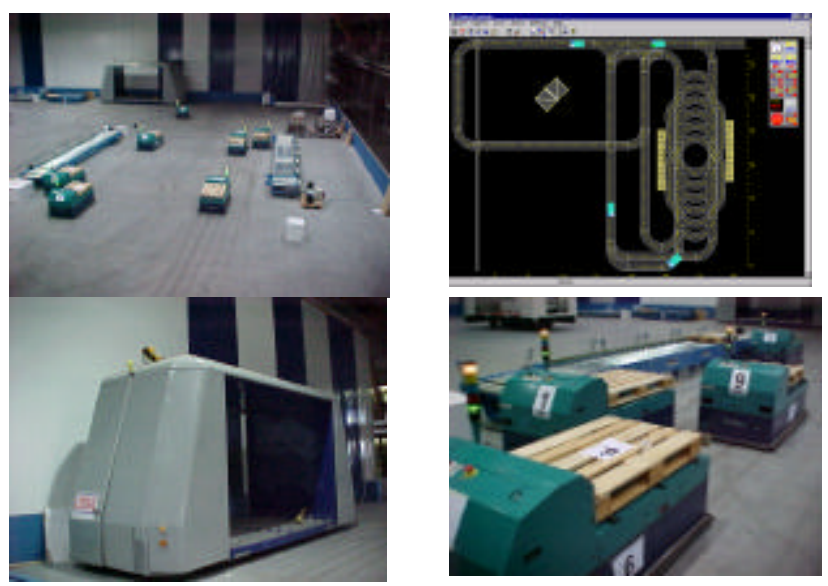

Figure 4. Overview of the TestSite

Figure 4 provides an overview of the TestSite. The scale models of the AGVs and automated material handling stations are shown on the top left. The scale models have a scale of 1:3, are 2 meter long, and weigh up to $750 \mathrm{~kg}$. The simulated control system can be seen at the top right corner. A full-scale prototype AGV is shown at the bottom left. This prototype AGV, manufactured by Lödige, has the following dimensions $6.6(1) \times 2.8(\mathrm{w}) \times 3.8(\mathrm{~h})$ meters, can weigh up to $11,000 \mathrm{~kg}$. At the bottom right four scale model AGVs and a scale model of a material handling station are depicted. 


\subsection{Combination of simulation, emulation, and prototyping}

In the fourth phase the combined experiments were conducted. In general 10 scale-model AGVs and several simulated AGVs were joined in one experiment. The scale models were used to test the vehicle dynamics, sensors, and batteries of the AGVs. To test the scalability and performance of the control system, several simulated AGVs were added to the experiments. Simulation offered possibities to test the scalability of the control systems, which is an important aspect of the ULS Schiphol. The ULS Schiphol will contain up-to 400 AGVs when it is fully implemented.

\section{Lessons learned}

In the SEP approach synchronization between simulation models, emulation models, and prototypes is critical. Two types of synchronization are distinguished; time and place (Versteegt \& Verbaeck 2002b). The synchronization of time is aimed at synchronizing the simulation clock of the simulated control system to the real-time internal clocks of the prototypes and emulated AGVs and material handling systems. Arena 4.0 RT and Simple++ 6.0 offer standard built-in features for real-time time progress in simulation models. For AutoMod 9.1 we constructed a 'wall-clock peeker'. Every fixed time unit, e.g. every tenth of a second, the wall-clock peeker synchronizes the simulation clock with to the internal clock of the computer. To synchronize the simulation models had to be slowed down. In some cases the simulated control system had sometime to 'catch-up' with the wall clock, especially when complex control algorithms had to be executed, e.g. collision avoidance. These are calculation intensive algorithms. The simulation model then lagged behind the wall clock and had to catch-up with the wall clock.

The synchronization of place proved to be more difficult, since there were various models of AGVs. First of all, there are the prototype AGVs. Secondly, there are the emulated AGVs. Finally, the simulation models contain two representations of the $\mathrm{AGVs}$, logic representation and the animation representation. All representations of the AGVs had to be synchronized. The synchronization between the representation of the AGVs in the simulation logic, which operated as the control system, and prototype AGVs is the most vital. When these two representations differ too much from each other, crashes between physical AGVs are bound to happen. The animation is allowed to run ahead or behind. The scalemodels and prototypes $\mathrm{AGVs}$ have, at this moment, no absolute coordinate tracking system to resolve their position. The AGVs are equipped with odometers, also called dead-reckoning systems, to keep track of their position. The AGVs use a magnetic grid in the floor for calibration of the odometers. These are, however, only relative calibrations. The odometers of the AGVs prove to work accurate enough to keep track of the actual positions of the AGVs. The movements of the AGVs are limited by virtual tracks. The parameters of the virtual tracks are given to the AGVs by the simulation model. The AGVs themselves calculate the virtual that needs to be followed to reach the end of the virtual track.

The communication is constructed in such a way, that the simulated control system does not see any differences between simulated, emulated, and prototype logistic resources. The simulated control system uses the same commands and protocol to control all types of logistic resources and receives back the same event notifications.

The prototype AGVs and material handling stations were used to evaluate the earlier developed simulation models. In earlier phases the simulation models were validated by structured walk-through by experts. The 'ultimate' validation, however, was performed by comparing the behavior of the simulated and the prototype AGVs and material handling stations.

By using the TestSite and the prototype AGVs the ULS Schiphol project became more concrete. When a largescale intelligent logistic or transport system is made more concrete research is stimulated and it becomes easier to mobilize decision makers around a project (Latour 1996).

\section{Conclusions}

The SEP approach was applied successfully at the TestSite. The SEP approach provides a 'rich' testing environment for automated logistic systems and control systems. Several aspects of the control systems and logistic resources can be tested before they are implemented. This improves the start-up or ramp-up phase of automated logistic systems. Within the SEP approach uncertainties on technology, control, and communication are solved at the beginning in simulation and emulation models. This strategy of first solving uncertainties and investing in logistic resources in later phases reduces the risks of investing in wrong technology for AGVs and material handling stations.

The SEP approach combines the advantages that the individual modeling techniques offer. In a fully simulated environment the control logic and assumptions on the logistic resources can be tested. Simulation provides a faster than real-time, safe environment, and researchers have good control over experiments. Emulation models can be used to debug software within logistic resources and to test communication aspects. The prototypes and scale models are used to test the technical aspects of the logistic resources. The combined experiments of the 
simulated, emulated, and prototype logistic resources provides a 'rich' environment for the evaluation of control systems and automated logistic systems. Several aspects of the control systems and logistic esources can be evaluated simultaneously, e.g. communication, scalability, and vehicle dynamics.

\section{References}

[1] AESOP. 1999. SiMPLE++ Reference Manual version 6.0, Aesop Corporation, Stuttgart, Germany.

[2] Auinger, F., Vorderwinkler, M., Buchtela, G. (1999). Interface driven domain-independent modelling architecture for "soft-commissioning" and "reality in the loop", in: Farrington, P.A., Nembhard, H.B., Sturrock, D.T., Evans, G.W. (eds.), Proceedings of the 1999 Winter Simulation Conference, IEEE, pp.798-805.

[3] Banks, J. 2000. Getting started with AutoMod, AutoSimulation, Bountiful, Utah.

[4] Banks, J. (1998). Handbook of simulation; principles, methodology, advances, applications, and practice, Wiley \& Sons.

[5] Ben-Ari, M. (1990). Principles of Concurrent and Distributed Programming, Prentice Hall, Upper Saddle River, New Jersey.

[6] Kelton, W.D., R.P. Sadowski, and D.A. Sadowski. 1998. Simulation with Arena, McGraw-Hill.

[7] Latour, B. (1996). Aramis or the love for technology, Harvard University Press.

[8] McGregor, I. (2002). The relationship between simulation and emulation, in: Yücesan, E., Chen, C.H., Charnes, J.M. (eds.), Proceedings of the 2002 Winter Simulation Conference, pp.1659-1666.

[9] Meer R. van der. (2000). Operational control of internal transport, doctoral dissertation, TRAIL Research School, Delft, the Netherlands.

[10] Mueller, G. (2001). Using emulation to reduce commissioning costs on a high speed bottling line, in: Peters, B.A., Smith, J.S., Medeiros, D.J., Rohrer, M.W. (eds.), Proceedings of the 2001 Winter Simulation Conference.

[11] Müller, T. (1983). Automated Guided Vehicles, Springer-Verlag.
[12] Pielage, B.A. (2001). Underground Freight Transportation. A new development for automated freight transportation systems in the Netherlands, 2001 IEEE Intelligent Transportation Systems Conference Proceedings, pp. 764-769, Oakland (CA), USA, August 25-29, 2001.

[13] Pielage, B.A. (2000), Design approach and prototyping of automated underground freight transportation systems in the Netherlands, $2^{\text {nd }}$ International Symposium on Underground Freight Transportation by Capsule Pipelines and Other Tube/Tunnel Systems (ISUFT), Delft, the Netherlands.

[14] Pyle, I., Hruschka, P., Lissandre, M. (1993). Real-time systems; investigating industrial practice, John Wiley.

[15] Schiess, C. (2001). Emulation: debug it in the lab-not on the floor, in: Peters, B.A., Smith, J.S., Medeiros, D.J., Rohrer, M.W. (eds.), Proceedings of the 2001 Winter Simulation Conference, pp.1463-1465.

[16] Verbraeck, A., Versteegt, C. (2001). Logistic for fully automated large-scale freight transport systems, 2001 IEEE Intelligent Transportation Systems Proceedings, pp. 774-779, Oakland (CA), USA.

[17] Versteegt, C., Verbraeck A. (2002a). Holonic control of large-scale automated logistic systems, The IEEE $5^{\text {th }}$ International Conference on Intelligent Transportation Systems, Singapore.

[18] Versteegt, C., Verbraeck A. (2002b). The extended use of simulation in evaluating real-time control systems of AGVs and automated material handling systems, in: Yücesan, E., Chen, C.H., Charnes, J.M. (eds.), Proceedings of the 2002 Winter Simulation Conference, pp.1659-1666. 